\title{
La reforma del Estado en Colombia: entre el ajuste fiscal y el fortalecimiento de la participación ciudadana $^{1}$
}

Rosalía Correa Young ${ }^{2}$

Recibido: Diciembre de 2005

Este artículo es producto de las reflexiones generadas en el marco del proyecto de investigación "La incidencia del Concejo de Santiago de Cali en la gobernabilidad democrática de la ciudad. El proyecto se inscribe en el Grupo de Investigación "Política Local, Nacional e Internacional; procesos, actores e instituciones, de la Pontificia Universidad Javeriana.

2 Socióloga. Profesora del Departamento de Ciencia Jurídica y Política de la Pontificia Universidad Javeriana de Cali y Coordinadora del Programa de seguimiento a la gestión del Concejo Municipal de Santiago de Cali - Cali Visible.

Aceptado: Abril de 2006 


\section{Introducción}

La Descentralización o Reforma del Estado en Colombia, cumple 20 años de vigencia. Su proceso ha oscilado entre la democratización de lo público y la aplicación del Consenso de Washington. Este artículo pretende dilucidar, a la luz de los paradigmas que la promueven, a cuál le ha apostado con mayor énfasis: al paradigma económico que promueve el libre juego de las fuerzas del mercado para la gestión de lo público y/o al paradigma sociopolítico que encuentra en la reforma una oportunidad política para que los sectores excluidos de la participación accedan a los derechos sociales: salud, educación, vivienda, servicios públicos, recreación.

El artículo se divide en tres partes. Un primer capítulo explica conceptualmente, los dos paradigmas. El segundo y tercer capítulo se refieren a su aplicación en Colombia. Finalmente, en las conclusiones se trata de mostrar, a manera de balance, el peso de cada uno.

\section{Marco conceptual: paradigmas de la reforma del Estado y la descentralización}

Para superar la crisis del modelo de Estado de Bienestar, cuando este no pudo responder a la magnitud de demandas sociales en bienes y servicios, los teóricos contemporáneos de las teorías económicas de la elección racional $^{3}$ argumentaron que la crisis se debía al peso de las políticas redistributivas y al crecimiento excesivo de la burocracia, "pervertía el orden democrático fundamental, basado en el individuo libre, la propiedad y los mecanismos del mercado" (Meny I, 1989, pg. 37). Por esa razón, era necesario descentralizar a nivel local o regional los procesos de política pública que contribuyen al desarrollo y, ceder al mercado aquellos servicios públicos ${ }^{4}$ que por su magnitud o por la limitación de los recursos, el Estado no era capaz de responder.

Por su parte, la óptica de sociólogos y politólogos encuentra en la descentralización, la posibilidad de una mayor integración social para la apropiación de los derechos sociales. Estos que fueron propiciados por el Estado de Bienestar y que pasan de acuerdo a los economistas a manos del mercado, encontrarían, en la descentralización, estrategias de gestión de políticas públicas alternativas: de corresponsabilidad mercado -sociedad civil-Estado.

Nos encontramos frente a dos paradigmas que promueven la reforma del Estado: el sociopolítico que podría definirse como una reforma policéntrica y el de la economía neoclásica como

3 Entre los cuales se encuentran los economistas Friedrich A. Hayek, Milton y Rose Friedman, Ostrom, entre otros

4 Los servicios públicos son aquellos que por su razón de ser no son bienes transables en el mercado, son indivisibles y están disponibles para todos 
mercadocéntrica. La primera encuentra fortalezas para la democratización, la equidad, la superación de los desequilibrios regionales y por ende la inclusión social; la segunda, el posicionamiento de las lógicas del mercado con la reducción, o desaparición de la intervención del Estado en aquellas funciones que los privados pueden resolver más eficientemente. Unos y otros colocan a la sociedad civil en un protagonismo activo, sea este político, en su relación con el Estado; sea económico, en su relación con el mercado, pero ambos la conciben como actor de primera línea.

Jordi Borja (1987) se ubica en la línea policéntrica; concibe la reforma como una posibilidad para el fortalecimiento de la democracia porque abre canales de intervención ciudadana en el proceso de la política pública y acerca, en el nivel local, el Estado al ciudadano. Sostiene que el Estado centralizado demostró su ineficiencia en la ejecución de políticas sociales y limitó la inclusión y participación política de diferentes sectores sociales. "La construcción del Estado democrático liberal se desarrolló, principalmente, a través de la extensión del sufragio universal y del papel de los partidos, en el marco del Estado - Nación y no en ámbitos locales o regionales". Según él, sólo a nivel local y con base en un proceso de descentralización se fortalece la autogestión social, las libertades individuales, la solidaridad, la innovación y la eficacia de lo público.

Los defensores del paradigma mercadocéntrico se centran en los comportamientos sociales, cuando de la escogencia de lo público se trata (public choice): "los individuos se comportan y toman sus decisiones de manera racional, únicamente en consideración a sus intereses personales y buscando optimizar los beneficios de sus decisiones" (Meny; 1992 , pg. 42). Por esa línea, el Estado no esta llamado a intervenir directamente en el suministro de bienes públicos, porque el mercado se encarga de ello y el Estado es el garante de las libertades individuales para que el mercado fluya.

Quienes critican la reforma estatal señalan que el proceso, en América Latina, está jalonado por los intereses monetaristas de los organismos internacionales: Fondo Monetario Internacional, Banco Mundial, Banco Interamericano de Desarrollo y gobiernos centrales que han condicionado sus aportes, ayudas o créditos a políticas de ajuste estructural ${ }^{5}$. No obstante,

$5 \quad$ Estas políticas están contempladas en el llamado Consenso de Washington. Con base en él los países latinoamericanos fueron conminados por las instituciones financieras de Washington a establecer políticas monetaristas, de mercado, abiertas, no intervencionistas. La situación de la deuda externa y la imperante necesidad de crédito, le dio a Washington el poder para lograr un cambio general en las políticas de la región y en buena parte del mundo en desarrollo. En Stewart Frances, 1998. Todos Entran. Propuesta para sociedades incluyentes. Editorial Santillana. 
José Luis Coraggio (1997) advierte que la propuesta sociopolítica tiene un importante reto para contrarrestar los impactos negativos de la reforma: impulsar cambios culturales para superar el clientelismo y la mercantilización de la política; propiciar espacios de participación ciudadana directa en el proceso de las políticas públicas; animar la cogestión de políticas: Estado -Sociedad- Mercado. Y cuando las fuerzas del mercado impongan la privatización de empresas estatales, luchar por su traspaso a colectivos de trabajadores u otra alternativa.

El reto esta puesto en el fortalecimiento de dinámicas de integración, lo que exige un esfuerzo de diferentes fuerzas políticas y sociales y de los gobiernos locales para formar, fortalecer y consolidar una ciudadanía consciente de sus derechos y no meramente reivindicatoria. Se trata de superar los mecanismos tradicionales de la democracia representativa y abrir canales para una relación más directa sociedad - Estado. Democracia en esta línea, quiere decir deliberación, reflexión y conocimiento de los problemas sociales y de lo que hay que hacer en materia de política pública, reconociendo las limitaciones de la acción gubernamental.

En América Latina, la ampliación de la democracia parece estar asociada a la participación ciudadana directa; es decir, a integrar en los procesos de desarrollo a una sociedad dividida y con grandes desigualdades, marginalidad y exclusión. En ese marco, la participación ciudadana es entendida como la forma de ampliar el campo de lo público hacia esferas de la sociedad civil o como interacción expresa entre Estado y Sociedad. Respecto de la primera, es una estrategia que busca que lo público no se agote en lo estatal e implica la transferencia a la sociedad civil, de funciones o decisiones que habían permanecido tradicionalmente en manos del Estado ${ }^{6}$. Respecto de la segunda, la sociedad civil "penetra" en el Estado y se convierte en contrapeso de decisiones estatales o en expresión y defensa de intereses de grupos o zonas territoriales. Para Nuria Cunill (1991), el eje lo constituye este tipo de práctica social que supone la interacción expresa Estado y sociedad.

Para una mayor acotación, el campo de la participación ciudadana en su relación directa con el Estado está en dos niveles: lo político propiamente y la gestión pública. En lo político, la ciudadanía participa en la toma de decisiones para incidir sobre el régimen o para decidir sobre las políticas públicas; en la gestión pública, interviene en la ejecución de acciones y en fiscalizar los procesos de ejecución. En el primer nivel, los actores sociales comparten el poder de decisión; en el segundo toman parte en la prestación de un servicio o en fiscalizar su ejecución

6 La prestación de ciertos servicios públicos como la salud, por parte de cooperativas $\mathrm{u}$ organizaciones de base que asumen responsabilidades para administrar y ejecutar recursos estatales y privados 
para corregirla o reorientarla. En este nivel, el poder político de la sociedad civil se ve limitado por las decisiones tomadas previamente.

En síntesis, el proceso de descentralización enfrenta dos caminos: el fortalecimiento de la ciudadanía y la ampliación de canales de participación para incidir de manera directa en el espacio público y/o, el traspaso de las funciones del Estado a las leyes del mercado. Cuál haga mayor presencia, o incline la balanza a su favor, no sólo depende de las normas vigentes, sino y fundamentalmente de la voluntad de las distintas fuerzas que hacen presencia a nivel local.

A renglón seguido se tratará la dinámica, respecto de la implantación de dos paradigmas, en Colombia y a partir de los años ochenta, cuando estableció medidas de descentralización administrativa, política y fiscal.

\section{El ajuste estructural}

En Colombia, la reforma del Estado se ha ido cumpliendo en un ambiente de tensión entre la ampliación de los canales de participación e intervención ciudadana, la lógica del mercado y una tendencia a la mercantilización de lo público. Comenzó como respuesta a la crisis de legitimidad y gobernabilidad que enfrentaba el Estado: ${ }^{7}$ movilizaciones, paros cívicos como expresión de inconformidad por promesas electorales incumplidas frente a demandas en servicios públicos, vías, educación, medio ambiente ${ }^{8} \mathrm{y}$ también por la presión del movimiento insurgente.

Una segunda fase surge con la Constitución de 1991. Esta coyuntura, que aunque respondía a la desestabilización institucional que persistía y había tomado nuevos visos con la presión del narcotráfico, también busca-

7 Reformas a la Constitución de 1886: Acto Legislativo 01 que introdujo la elección popular de alcaldes y las Ley 11 y 12 de 1986 entregaron a los municipios, la administración de las políticas en salud, educación, servicios públicos, vivienda, deporte, asistencia técnica agropecuaria, etc., fortalecieron los fiscos con recursos propios y transferencias de la nación. De igual manera, establecieron canales de participación ciudadana en los asuntos públicos: juntas administradoras locales como espacio de representación los intereses territoriales; juntas directivas de los establecimientos públicos y de las empresas industriales o comerciales encargados de la prestación directa de los servicios públicos; celebración de convenios, contratos o acuerdos entre juntas de acción comunal, organizaciones no gubernamentales y municipios para la ejecución de obras o funciones de carácter público; consultas populares para decidir sobre asuntos de interés ciudadano.

8 Entre 97 y 1985 se realizaron 300 paros cívicos en Colombia (cifras de Pedro Santana, Revista Foro, 1983). 
ba adecuar la estructura estatal a las exigencias del modelo mercadocéntrico: reducción de su tamaño, privatizaciones, desregulación de la economía, despolitización y tecnocratización de la gestión pública ${ }^{9}$. En ese marco se reglamentó la libre competencia en la prestación de servicios públicos; se desmontaron instituciones financieras; se vendieron total o parcialmente empresas estatales; se liquidaron instituciones de orden nacional.

Hay una tercera fase que es la del ajuste fiscal; lo impone, en el año 2000, el Fondo Monetario Internacional, para reducir el déficit fiscal nacional que en ese momento ascendía al $28.4 \%$ del PIB y representaba en intereses, el 4\% del PIB y la cuarta parte de los gastos (J. Castro, 2001). Como estrategia, el Fondo exigió el recorte de las transferencias ${ }^{10}$ a las entidades territoriales contando con el respaldo de la tecno- cracia nacional que consideraba excesivo el tratamiento dado por los constituyentes de $1991^{11 .}$

Algunas razones que explican el déficit fiscal departamental y municipal son: la ausencia de políticas nacionales, permanentes y no coyunturales para el fortalecimiento de la descentralización y la irresponsabilidad propia de los gobernantes locales. Las políticas nacionales para el fortalecimiento institucional territorial, iniciadas en el primer periodo de los años noventa, no continuaron; los municipios quedaron a su suerte y muchos no poseían capacidades técnicas para enfrentar con éxito una tarea que desconocían administrativa, financiera y legalmente. La Ley 358 de 1997, formulada para medir la capacidad de pago de las entidades territoriales para efectos de endeudamiento, llegó cuando municipios y departamentos enfrenta-

$9 \quad$ El énfasis mercadocéntrico se advierte en las leyes aprobadas para el manejo de la salud (ley 100/1993), los servicios públicos (ley 142/1994) la educación (115/...), entre otras.

10 El régimen de transferencias se inició con el traslado del impuesto nacional a las ventas (1963) y se consolidó con la ley 12 de 1986, que estableció la participación de los municipios en ese impuesto: $30.5 \%$ en el primer año, hasta completar el $50 \%$ en 1992. La Constitución de 1991 dispuso que el situado fiscal -transferencia que se hacía a los departamentos para gastos en salud y educación-, se liquidara con base en los ingresos corrientes de la nación; y la ley 60 de 1993 reglamentó que se incrementara entre el 19\% en 1993 hasta el 24.5\%. La participación de los municipios también recibió ese enfoque: $14 \%$ en 1993 hasta el $22 \%$ en el 2002

11 Eduardo Wiesner (1995), en su libro la Descentralización, el Gasto social y la Gobernabilidad en Colombia, publicado por el Departamento Nacional de Planeación y la Asociación Nacional de Instituciones Financieras hace una crítica a la descentralización físcal y señala que la mayoría de los municipios padece de "pereza fiscal" sus recaudos por concepto de rentas propias no financian sus gastos de funcionamiento.....Y cada vez más "hay una creciente de los recursos de la Nación" (pg25). 
ban un super endeudamiento y pagaban tasas de interés del DTF ${ }^{12}+10$.

El afán de muchos gobiernos por cumplir compromisos de campaña y reducir el déficit de obras públicas en sus regiones, los llevó a tocar las puertas del sistema financiero, máxime si éste no esperaba que le solicitaran los créditos sino que los ofrecía a manos llenas con la creencia de que el "Estado no se quiebra" (Castro, J. 2001). Por otra parte, la cultura política tradicional se apropió de las regiones contribuyendo a la malversación y privatización de fondos públicos, a la excesiva burocratización de las administraciones, concejos, asambleas, contralorías y personerías departamentales y municipales; como también, para establecer prebendas salariales y prestacionales, concertadas mediante convención colectiva. A esto se sumó la recesión económica que a mediados de los noventa afectó la industria y los valores de la propiedad inmueble; y con la caída de los precios de la propiedad, el Gobierno ${ }^{13}$ congeló los avalúos catastrales, afectando el ingreso de los municipios.
La crisis financiera, además de convertirse en un argumento para reducir las transferencias, fue aprovechada para imponer ajustes a las entidades territoriales respecto al uso de sus ingresos propios. El Gobierno Nacional las incluyó en la ley 550 de 1999, y municipios y departamentos con cartera morosa podían solicitar una reestructuración de la deuda, sometiéndose a un proceso de intervención económica por parte de la nación, que designaba funcionarios de orden nacional para coadministrarlos.

Adicionalmente, el Congreso aprobó la ley 617 del 2000 para racionalizar el gasto de las entidades territoriales, categorizándolas con base en su población y sus ingresos corrientes de libre destinación. En esa ley se definieron los porcentajes máximos de gastos de funcionamiento, gastos de contralorías y personerías, y honorarios de concejales y diputados; de igual manera, la financiación y provisión del pasivo prestacional y pensional, con base en los ingresos corrientes de libre destinación ${ }^{14}$.

12 DTF: Depósitos a término del sistema financiero. Es el promedio de las tasas de interés, de las captaciones del sistema financiero. Para los años 94 al 95, el DTF llegó, aproximadamente al $30 \%$.

13 Ley 550 de reactivación económica.

14 Los ingresos corrientes de libre destinación son los ingresos corrientes excluidas las rentas de destinación específica, entendiendo por estas las destinadas por ley o acto administrativo a un fin determinado. Esta definición limita la financiación de los gastos de funcionamiento con recursos de: a) El situado fiscal; b) La participación de los municipios en los ingresos corrientes de la Nación de forzosa inversión; c) Los ingresos percibidos en favor de terceros que, por mandato legal o convencional, las entidades territoriales, estén encargadas de administrar, recaudar o ejecutar; d) Los recursos del balance, conformados por los saldos de apropiación financiados con recursos de destinación específica; e) Los recursos de cofinanciación; f) Las regalías 
El ajuste fiscal continuó con el Acto Legislativo 01 del 2001 que creó el Sistema General de Participaciones sustituyendo la legislación anterior ${ }^{15}$; una "bolsa común" de la que se hacen los giros y para su reparto se tiene en cuenta: población atendida y por atender; población urbana y rural, eficiencia administrativa y fiscal, y equidad, con base en el indicador del pobreza que distribuye el DANE ${ }^{16}$.

A renglón seguido, la ley 715 de 2001 reglamentó el Acto Legislativo en cuanto a las funciones que le corresponden a la nación, a los departamento $\mathrm{y}$ a los municipios en educación y salud, y en cuanto a la distribución de los recursos del Sistema General de
Participaciones para educación, salud y propósitos generales ${ }^{17}$.

Aunque las medidas tomadas buscaban disminuir el déficit fiscal nacional, controlar la crisis financiera y fortalecer la descentralización presionando el esfuerzo ${ }^{18}$ y la capacidad fiscal ${ }^{19}$ la dependencia de las transferencias nacionales para inversión sigue siendo alta y los recursos propios escasos $-11.49 \%$ en el $2003 ; 12.65 \%$ en el 2004 . Del total de la inversión municipal, en el 2003 , solo el $15 \%$ fue financiado con recursos propios, aunque las grandes ciudades aportaron un mayor porcentaje. Para quienes se acogieron a la ley 550 , sus recursos propios están destinados a amortizar la deuda. En los de-

y compensaciones; g) Las operaciones de crédito público, salvo las excepciones que se establezcan en las leyes especiales sobre la materia; h) Los activos, inversiones y rentas titularizadas, así como el producto de los procesos de titularización; i) La sobretasa al ACPM; j) El producto de la venta de activos fijos; k) Otros aportes y transferencias con destinación específica o de carácter transitorio; 1) Los rendimientos financieros producto de rentas de destinación específica (Tomado de la Ley 617/ 2000, art. 3).

15 Ley 60 de 1993.

16 DANE. Departamento Administrativo Nacional de Estadística.

17 Del total de los recursos que conforman el sistema, se deduce el $4 \%$ que se distribuye así: $0.52 \%$ para los resguardos indígenas, el $0.08 \%$ entre los municipios cuyos territorios limiten con el Río Grande de la Magdalena en proporción a la ribera de cada municipio, según la certificación del Instituto Geográfico Agustín Codazzi, el 0.5\% a los distritos y municipios para programas de alimentación escolar y el 2.9\% al Fondo Nacional de Pensiones de las Entidades Territoriales, Fonpet, Una vez realizada esta deducción, a educación le corresponde el $58.5 \%$, a salud el $24.5 \%$ y, para propósitos generales (promoción del empleo, restaurantes escolares, atención a la población vulnerable, entre otros) el $17.0 \%$.

18 Esfuerzo fiscal: voluntad política del gobernante para cumplir metas de recaudo presupuestadas, controlar exenciones, y lograr la voluntad de los gobernados para pagar oportunamente, sin evasión ni elusión.

19 Aquella referida a la habilidad de los gobernantes para incrementar sus recursos propios determinados por la estructura económica de la entidad territorial; como también, la disponibilidad de recursos imponibles o bases gravables 
partamentos, las condiciones son más precarias: los ingresos propios entre el 2003 y el 2004 descendieron, en promedio, del $21.25 \%$ al 19.33 , generando mayor dependencia de las transferencias, como también menor capacidad fiscal para inversión propia.

$\mathrm{Y}$ aunque es importante que los municipios eleven su capacidad fiscal: Incremento de recursos propios con base en actualizaciones catastrales y alza de las tarifas sobre las que se cobran los impuestos municipales. Los estudios del DNP muestran una capacidad fiscal baja: "la tarifa efectiva promedio que cobra el $77 \%$ de los municipios por impuesto predial está por debajo del 4 por mil, mientras la tarifa de las ciudades grandes oscila entre $1.1 \mathrm{y}$ 8.4 por mil, siendo Medellín, Cali, Barranquilla y Envigado las ciudades con la tarifa más alta dentro de las cuarenta ciudades grandes".

Un resultado del ajuste fiscal ha sido la reducción de la planta de cargos y de funciones administrativas, profesionales u operativas que venían realizando los municipios directamente y que representaban significativas erogaciones en materia salarial y prestacional. Como solución, esta la ley 80 de contratación a particulares, como también los outsourcing. Con base en ello proliferan empresas asociativas de trabajo; cooperativas, fundaciones, etc., a quienes los municipios o departamentos contratan para la prestación de servicios docentes, medico-asistenciales, barrido de calles, recolección de basuras y hasta el recaudo de los impuestos $^{20}$. Esta solución, propia de un modelo de Estado que se propuso reducir su tamaño para ceder funciones a las fuerzas del mercado ha sido capitalizada, en Colombia, por la política tradicional que estimula su creación y conservar clientelas y apropiar dineros públicos de manera irregular ${ }^{21}$.

En consecuencia, el desmonte del Estado benefactor y el advenimiento del paradigma mercadocéntrico instauró prácticas mercantilistas con recursos públicos (Varela, 2003), que no se caracterizan propiamente, por las bondades que el paradigma promulga. Entre quienes las realizan se encuentran organizaciones, empresas o personas sin el profesionalismo adecuado para asu-

20 La Administración del Municipio de Cali parece ser la más avanzada en este tipo de contrato. Contrató a la firma Sicali para el recaudo de los impuestos locales. Y a pesar de las denuncias y debates suscitados, la contratación, aparentemente no tiene vicios de forma y cumple con los requisitos de la ley de contratación.

21 Esta costumbre, parece haberse desbordado en Cali. La contratación de organizaciones privadas para la ampliación de cobertura de la educación primaria y secundaria ha sufrido un duro revés. Un número importante de organizaciones contratadas no reúnen los requisitos exigidos de idoneidad o capacidad en infraestructura educativa y en cuerpo docente. Por esa razón, dos concejales de la ciudad se encuentran presos y hay unos más, cuyas conductas son objeto de investigación porque aparecen relacionados con empresas que prestan servicios de barrido de calles y mantenimiento de zonas verdes (www.elpais-cali.terra.com.co.) 
mir responsabilidades en educación, salud, entre otras; que tienen como propósito, la obtención de dinero, por todos los medios, comercializando con el déficit en derechos sociales que tiene un amplio sector de la población colombiana sin oportunidades para acceder a la escogencia pública (public choice). En esa línea, el proceso de descentralización puede terminar dedicándose a contratar, con los recursos de las transferencias nacionales, a organizaciones del más disímil carácter, para que sean ellas las ejecutoras de la política pública. Con base en ello, la brecha entre quienes tienen oportunidad para escoger libremente las ofertas del mercado relacionadas con políticas sociales y los que no la tienen, puede llegar a ser mayor y el Estado que ha tratado de disminuir la deuda social, entregando a los particulares la hechura de las políticas, no cuenta cualitativa ni cuantitativamente con capital humano para garantizar, calidad en la prestación de los servicios ni el control fiscal y político requeridos.

De este recorrido se coligen varios aspectos. En primer lugar, la nación ha reducido las transferencias y al exigir esfuerzo y capacidad fiscal deja al libre juego de las fuerzas del mercado a municipios y departamentos. Si bien, los puso en cintura frente al desmán financiero, el esfuerzo fiscal no representa montos considerables para asumir la prestación de servicios; y la capacidad fiscal está limitada en el $80 \%$ de los municipios por sus condiciones sociales y de economía rural. Son los municipios que tienden a desaparecer como tales ${ }^{22}$.

En el 20\% restante se encuentran las grandes ciudades y los medianos municipios, que son los receptores de la población rural desplazada; enfrentan no solo sus debilidades políticas, sociales y económicas estructurales, no resueltas con la descentralización, sino, además, las coyunturales: incremento de la violencia urbana, desorden, polución, contaminación, pobreza, desempleo.

En segundo lugar, y debido a la cultura política tradicional, la reforma del Estado ha tomado un rumbo peligroso en cuanto a la gobernabilidad y a la legitimidad del Estado, pues la figura de la contratación como medida para reducir costos, ha mercantilizado el servicio público y se ha convertido en una nueva estrategia para la reproducción de costumbres clientelistas y para continuar subordinando sectores excluidos de las oportunidades que brindan las leyes del mercado y la competencia.

En síntesis, el proceso refleja una tendencia hacia el desmonte del Estado; a la reducción de las entidades territoriales por sustracción de materia; a la privatización de lo público que no escapa a una lógica de aprovechamiento y apropiación indebida de recursos públicos.

22 El artículo 20 de la ley $617 / 2000$ contempla la alternativa de fusión de municipios que no logren demostrar viabilidad financiera, de acuerdo a los límites establecidos para gastos de funcionamiento, honorarios de concejales y topes de ingresos propios. 


\section{El fortalecimiento de la democracia}

El fortalecimiento de la democracia es el otro ángulo desde donde se promueve la reforma del Estado y aparece como posibilidad la inclusión política de amplios sectores de la población con base en la apertura de una serie de canales de participación, más allá de los electorales. En Colombia, que en sus primeros pasos estableció la elección popular de alcaldes, reglamentó canales de participación directa, y la Constitución de 1991 declaró como principio fundamental "la democracia representativa y participativa". Este paso ha significado, al menos en términos formales, la proliferación de formas de participación a nivel local, departamental y nacional: electoral (alcaldes, gobernadores, juntas administradoras locales), política (plebiscitos, referéndum, consulta popular, revocatoria del mandato de alcaldes y gobernadores), administrativas (juntas directivas, consejos o comités de dependencias y entidades públicas, juntas de acción comunal, de vivienda comunitaria, juntas administradoras locales), de planificación (consejos territoriales de planeación y de desarrollo rural), de control social ( asociaciones de usuarios de los servicios en salud y educación, veedurías ciudadanas).

Además, existen canales informales que no están explícitamente reglamentados por la legislación nacional: organizaciones, asociaciones, comités; en síntesis, ONG que se crean con objetivos sociales, culturales, comunitarios, de género, de étnia, jurídicos, para promoción, para realizar planes de capacitación, de bienestar, de recuperación del medio ambiente, etc., y que han sido reconocidas, por el Decreto 0427 de 1996, como personas jurídicas sin ánimo de lucro.

La elección popular de alcaldes y gobernadores se han movido entre el surgimiento de nuevas fuerzas políticas, en ciertos grandes y medianos centros urbanos ${ }^{23}$ y el bipartidismo. No obstante, muchas de ellas se escudan en diferentes nominaciones para atraer al electorado ${ }^{24}$. Por su parte, liberales y conservadores, en la última contien-

23 Un caso representativo de esta incipiente tendencia, es Bogotá que en tres ocasiones ha elegido candidatos independientes y para el 2004 eligió un candidato de izquierda democrática con el $46 \%$ de la votación y una abstención del 54\%. Medellín también sorprendió para estas elecciones con un alcalde independiente. Elecciones anteriores en municipios intermedios como Pasto, Manizales y Pereira, también han elegido alcaldes independientes. Y Darío Restrepo (2005) en su artículo "contribución de la descentralización a la superación de la crisis económica y política", en una nota a pie de página señala que entre el $11 \%$ y el $35 \%$ de las alcaldías y gobernaciones han estado en manos, desde la primera a la última elección, de agrupaciones distintas a los beneficiarios del Frente Nacional, los partidos Liberal y Conservador.

24 El movimiento Equipo Colombia (en 65 municipios), Cambio Radical (en 25 municipios). Muchos de sus miembros pertenecen al liberalismo o conservatismo pero hoy 
da electoral -2003- estuvieron representados en el 35\% de los municipios del país. En los pequeños municipios, el narcotráfico, los paramilitares o el movimiento guerrillero han bloqueado o manipulado para su beneficio económico o político, este canal de participación electoral.

En ese sentido la democracia representativa $^{25}$, sigue siendo débil; no muestra avances que trascienda la simple demanda, la cultura utilitarista ligada a intereses clientelistas, caudillistas y personalistas, o la indiferencia por los asuntos públicos. La participación electoral, desde la primera elección de alcaldes, no incrementa su cualificación y el programa de gobierno no es, en términos generales, el elemento que convoca. El electorado sigue cautivo de una clase política que se amaño en el poder y no hay registro de revocatoria del mandato por incumplimientos al plan de desarrollo, corrupción o denuncias comprobadas ${ }^{26}$.
El municipio de Cartagena, por ejemplo, acaba de elegir con 54.584 votos y una abstención del 78\%, al ex alcalde Nicolás Curi, quien en 1.999 fue obligado a abandonar el cargo acusado de corrupción. Al otro lado de Colombia, en Cali, el alcalde Apolinar Salcedo y la ciudad, atraviesan por el peor momento. Investigaciones sobre corrupción, parcelación de dependencias administrativas entre los concejales de la coalición, contrataciones indebidas para recaudar los impuestos municipales o para inversión con los recursos del plan de Ampliación de Cobertura en Educación o para la recolección de basuras. Y aunque alguna parte de la ciudadanía ha promovido la revocatoria del mandato, no hay muestras de un rechazo generalizado; por el contrario hay sectores que se oponen con argumentos de tipo clasista. Adicionalmente, el proceso enfrenta limitaciones contempladas en la ley que lo hacen dispendioso, oneroso y poco viable ${ }^{27}$.

se encuentran,"coyunturalmente" por fuera, apoyando el gobierno del Presidente Álvaro Uribe Vélez; algunos intentan fortalecer su movimiento o conformar un nuevo partido: el Partido de la "U".

25 Ver Velásquez, Fabio y González, Esperanza. “QQué ha pasado con la participación ciudadana en Colombia?”. Bogotá, Fundación Corona, 2003.

26 La revocatoria del mandato, pese a que ha sido promovida en distintos municipios, no ha procedido porque su reglamentación en la práctica la hace inaplicable. Con base en la Registraduría del Estado Civil, entre los años 1999 y 2000 se promovieron seis revocatorias de mandato y ninguna fue exitosa.

27 El proceso de revocatoria del mandato se realiza en dos momentos. Un primer momento en el cual se hace una "solicitud de convocatoria a pronunciamiento popular para revocatoria, mediante un memorial que suscriben los ciudadanos en número no inferior al $40 \%$ del total de votos que obtuvo el elegido". Adicionalmente, esta solicitud debe ser ratificada en un proceso electoral, "por la mitad más uno de los votos ciudadanos que participen en el, siempre que el número de sufragios no sea inferior al cincuenta y cinco por ciento $(55 \%)$ de la votación válida registrada el día en que se eligió al respectivo mandatario" (Ley 741 de mayo del 2002 que reformó la ley 131 y 134 de 1994). 
El tema de la participación no cuenta con evaluaciones sistemáticas o parciales sobre los impactos que ha tenido la proliferación de estos espacios en el mejoramiento de la inclusión o de la formación y cualificación sociopolítica para intervenir en procesos de decisión o para evaluar decisiones y acciones. El espacio más relevante sigue siendo el de las juntas de acción comunal creadas en $1958^{28}$. En Colombia existen aproximadamente 43.000 juntas con una afiliación de 4 millones de personas. Tienen un promedio de 40 a 70 miembros y contribuyen en obras de infraestructura barrial, promueven actos culturales y actividades de recreación y deporte. Recientemente están participando en la definición de planes de desarrollo local. Pero aunque su labor es importante y reconocida, funcionan como células base de las redes políticas de los partidos. Como contraprestación por "promesas de obras", avalan candidatos a alcaldías, concejos, asambleas, etc., y los concejales, principalmente, destinan recursos del presupuesto local para las obras de "sus" JAC, controlando así sus "cuotas" electorales (Ceballos, 2001).
La reforma del estado y en concreto la ampliación de canales de participación han permitido que muchos líderes de acción comunal reorienten sus actividades o se reproduzcan en diferentes organizaciones donde se ejerce la participación ciudadana. Es frecuente encontrarlos como veedores en consejos municipales o haciéndose elegir a juntas administradoras locales, o como miembros de los comités locales de salud. Varios de ellos hacen carrera, para un determinado movimiento político, logrando el reconocimiento de "su jefe", que lo o la promociona para acceder a una curul en los concejos municipales o en las asambleas departamentales.

Otros espacios de participación formal reconocidos por la ley, no han despertado la motivación y apropiación ciudadana. Unos aparecen más atractivos que otros, unos tienen más promoción que otros, unos encuentran más o menos voluntad política por parte de las autoridades encargadas de reglamentarlos y ponerlos en marcha. Las juntas administradoras locales, por ejemplo, que no son organizaciones comunitarias, pero fueron creadas por la ley 136 de 1994, como cor-

28 Artículo 103, 38, 49, 68 $, 105,107,270,311,318,329,339,340$ y 369 de la Constitución Política. Adicionalmente, la ley 134 de 1994 de mecanismos de participación, Ley 70 de 1993 de protección de las comunidades negras, Ley 99 de 1993 del Medio Ambiente, Ley 152 de 1994 del Plan de Desarrollo, Ley 136 de 1994 de Régimen Municipal, Ley 142 de 1994 de Servicios Públicos, Ley 397 de 1997 de Cultural, Ley 181 de 1995 de Fomento del Deporte, Ley 101 de 1993 de Desarrollo Agropecuario y Pesquero, Ley 454 de 1998 de Economía Solidaria, Ley 115 de 1994 General de Educación, Ley 100 de 1993 de Seguridad Social, Ley 375 de 1997 de Juventud, Ley 388 de 1997 o Plan de Ordenamiento Territorial, Ley 62 de 1993 de Seguridad, Convivencia y Paz, Ley 617 de 2000. 
poraciones públicas de elección popular. A través de ellas se trataba de modernizar el funcionamiento de los municipios, dividiéndolos en comunas, en las áreas urbanas y en corregimientos, en las áreas rurales. En cada una de esas subdivisiones se tendría una junta administradora local, como instancia de promoción y coordinación de la participación ciudadana, así como con funciones de planeación y presupuestación, y de vigilancia y control de las administraciones locales. Hoy existen en algunas grandes y medianas ciudades, porque su creación, depende legalmente de la voluntad política de los concejos municipales que en muchos municipios se han resistido a ceder o compartir parte de sus propias responsabilidades.

Además, de las juntas de acción comunal, como organizaciones de participación ciudadana directa, se destacan las veedurías ciudadanas, los consejos territoriales de planeación, las asociaciones de usuarios de salud, las juntas municipales de educación y las asociaciones de padres de familia.

La experiencia de estas instancias participativas no sólo es diferente en su actuación sino también en sus motivaciones y en los vínculos institu- cionales u organizacionales que animan sus procesos. Realizan su labor de manera voluntaria, ad honores; característica que les hace vulnerables para operar y tener objetivos de largo plazo relacionados con la representación de intereses colectivos.

A excepción de los consejos territoriales de planeación, las veedurías están conformadas por líderes comunitarios vinculados a las juntas de acción comunal que actúan en los barrios y veredas de los estratos bajo y medio bajo de la población ${ }^{29}$; son débiles conceptualmente en el conocimiento de lo público, de las funciones del Estado en sus distintos niveles y de sus mismas tareas. Se integran y desintegran de acuerdo al objetivo que los anima; que por lo general es coyuntural. En ocasiones cuentan con acompañamiento de instituciones u organizaciones de la sociedad civil que promueven su creación y les brindan soporte conceptual y técnico.

Las veedurías ciudadanas, las asociaciones de usuarios de salud, las asociaciones de padres de familia se conforman, principalmente, para hacer seguimiento a la ejecución de programas y proyectos: la construcción de una vía o de un acueducto rural, una

29 Un registro de información realizado por Foro Nacional por Colombia, sede Bogotá, con base en una muestra de veedores de la capital de la República, permitió establecer un perfil socioeconómico aproximado de ellos: se trata de individuos con un promedio de edad de 47 años, de estratos bajo y medio-bajo, con un promedio de cuatro personas a cargo, un grado de escolaridad medio o superior, una ocupación que le permite ingresos para sobrevivir y alguna vinculación a organizaciones locales. En Fabio E. Velásquez, La Veeduría Ciudadana en Colombia: en busca de nuevas relaciones entre el Estado y la Sociedad Civil. 
escuela, o a un contrato de prestación de servicios como los servicios de promoción y prevención en salud, programas de vacunación, de educación sexual y reproductiva, de lucha contra el cáncer, etc.

Hay otras veedurías con mayor nivel de formalidad: las organizaciones sociales, instituciones universitarias, fundaciones que cuentan con recursos financieros y humanos; con apoyo privado o de organismos internacionales que realizan ejercicios permanentes de control social. Varias de estas experiencias tienen alguna trayectoria y reconocimiento en la acción pública local. En esta modalidad se encuentran programas de seguimiento a la gestión de los concejos municipales de Cartagena, Bogotá, Barranquilla, Cali. Seguimiento a la gestión de los alcaldes de Cartagena, Bogotá, Medellín, Manizales, Cali.

A estas experiencias se añaden las obtenidas por organismos gubernamentales o no gubernamentales que promueven el desarrollo local y regional e incluyen la participación como eje de sus decisiones; son permanentes o transitorias, dependiendo de su objetivo, y algunas han sido reconocidas nacional e internacionalmente. Están lideradas por instituciones públicas del orden nacional como la Red de Solidaridad, el Programa Plante o las Zonas de Reserva Campesina; por instancias de orden departamental como la promoción de la participación ciudadana en el Departamento de Cundinamarca ${ }^{30} \mathrm{o}$ de orden municipal como el Municipio Saludable para la Paz en Versalles - Valle del Cauca. También hay experiencias promovidas por otras instituciones como la iglesia católica o por consorcios entre organismos públicos y privados: el Fondo para la Reconstrucción del Eje Cafetero (FOREC), el Programa de Desarrollo y Paz del Magdalena Medio (PDPMM) y la Corporación para el Desarrollo y la Paz (VALLENPAZ) $^{31}$.

Esta muestra de experiencias posee particularidades que las diferencia de otras modalidades de participación explicadas anteriormente. En primer lugar su liderazgo esta concentrado en instituciones públicas de nivel nacional o en organizaciones regionales sin ánimo de lucro, que han ganado aceptación y legitimidad social. En segundo lugar, en su mayoría integran la participación de la sociedad civil en las decisiones de los proyectos y programas que realizan, como en su ejecución y evaluación. En tercer lugar, las administraciones públicas de los territorios locales o regionales donde tienen su centro de operaciones no son protagonistas directas, más bien optan por permanecer al margen, cuan-

30 Este ejercicio fue liderado como parte fundamental del programa del gobernador Andrés González entre 1998 y 2000.

31 Se han señalado estas experiencias por su impacto y divulgación nacional. Es posible que existan otras, pero al respecto no se encontró documentación sistematizada. Ver anexo. 
do no se convierten en obstáculo para que los programas salgan adelante; la prevención, frente a las prácticas clientelistas y de la política tradicional, también ha contribuido a marginar la participación estatal en los territorios departamentales o locales ${ }^{32}$. En cuarto lugar, obtienen y manejan un presupuesto importante donde confluyen recursos provenientes del presupuesto nacional, de la empresa privada $\mathrm{u}$ organismos internacionales bajo la figura de préstamos o donaciones. Esta situación les da cierta holgura para desarrollar proyectos productivos, de capacitación, de desarrollo social, etc., que logran impactos positivos en las regiones donde laboran.

Un quinto elemento tiene que ver con el fortalecimiento de la participación ciudadana como resultado de esas experiencias. En ese sentido hay tres tipos de experiencias que arrojan diferentes resultados. Las experiencias de instituciones públicas como el FOREC, el Programa PLANTE o la Red de Solidaridad que son programas coyunturales, y en la mayor parte de las ocasiones, promovidos por el gobierno nacional y supeditados a los planes de desarrollo nacional, Estos programas aunque tratan de controlar las actitudes clientelistas no lo logran por las dinámicas propias de cada región y los intereses de los actores que hacen presencia. Adicionalmente están expuestos a la desconfianza y a la falta de credibilidad de las comunidades adonde llegan. Por una parte, porque incumplen las promesas en materia de recursos y por otra parte, porque dilatan la ejecución de los programa en las operaciones burocráticas del centro. Estas situaciones son menos evidentes en las experiencias de segundo tipo, aquellas que son lideradas por consorcios donde confluyen organismos públicos y/o privados: Desarrollo y Paz del Magdalena Medio y corporación para el Desarrollo y la Paz del Valle - Vallenpaz. Estas organizaciones, al lograr mayor credibilidad han impactado positivamente en el fortalecimiento y consolidación de procesos participativos permanentes.

El tercer tipo se refiere a experiencias de carácter local propias de un liderazgo comunitario. Es el caso del programa Municipio Saludable para la Paz. Surge en el municipio de Versalles, en el Valle del Cauca. Está ubicado a $234 \mathrm{~km}$ de Cali, en el piedemonte de la cordillera Occidental. Tiene aproximadamente 12.000 habitantes (4.800 en el área urbana y 7.200 en el área rural); en términos culturales es relativamente homogénea y se caracteriza por las raíces antioqueñas de sus habitantes ya que es una zona de colonización que se desarrolló con la extensión de la producción del café desde finales del siglo XIX.

Entre sus raíces culturales parece haber una tradición comunitaria y de

32 El FOREC fue muy criticado porque en muchas ocasiones actúo sin mayor integración con las administraciones locales generando una especie de gobierno, por encima de... 
trabajo colectivo: entre los colonos abrieron carretera, construyeron iglesia y conformaron cooperativas agrícolas y en 1947 crearon el Comité de Desarrollo Campesino. El Municipio también ha tenido problemas de violencia; en los años 40 y 50 a consecuencia de la confrontación bipartidista liberal - conservadora, y en los 80 , el narcotráfico. Sin embargo, parece ser que sus raíces han sido un terreno abonado para liderar y consolidar un proceso de participación comunitaria de origen local, con liderazgo local como el de Municipio Saludable para la Paz.

Comenzó en 1989 cuando por determinación del Ministerio de Salud ${ }^{33}$ los municipios debían crear los comités de participación comunitaria en Salud para fiscalizar la prestación de estos servicios. Con base en esa iniciativa, dos médicos del hospital local decidieron utilizar a fondo ese mecanismo y promover una serie de acciones con participación de la comunidad, con el apoyo de la iglesia católica. En 1994, y como parte de la Ley 100, el ministerio de Salud lanzó el programa de Empresas Solidarias de Salud en los municipios, pero Versalles por su tamaño, no podía crear una ESE; sin embargo, con el apoyo de organizaciones privadas como la fundación Carvajal y otras entidades, hizo posible que naciera la cooperativa Solidaria de Salud de Versalles (Coopsaver). De esta manera se buscó mejorar el acceso a los servicios de salud de la población estrato 1 y 2 y lograr una reducción en los precios de las medicinas y de los servicios médicos. Este ejercicio recibió asistencia técnica de la organización Panamericana de la Salud y en 1999 fue escogido como Municipio Saludable por la Paz.

La experiencia ha ido creciendo, y hoy, al interior del comité de Participación Comunitaria en Salud, que ha tenido constantes ajustes, existen siete subcomités: Salud, Desarrollo Educativo y Social, Desarrollo Agropecuario y Rural, Gestión Empresarial, Infraestructura y Servicios Públicos, Seguridad Ciudadana y Juvenil. Adicionalmente, ha dejado de ser un proceso desarrollado por voluntarios y se ha convertido en una organización generadora de opciones laborales, siendo el tercer generador de empleo del municipio.

De otro lado, las relaciones del Comité con las administraciones municipales y el concejo han sido cambiantes, dependiendo de los intereses políticos del alcalde de turno y de los concejales. En principio, el Comité fue visto con sospecha; cuando el proceso comenzó a tomar auge y a recibir atención nacional e internacional, la alcaldía comenzó a interesarse, pero los miembros del Concejo Municipal optaron por una actitud de oposición, dado que el programa no compartía lógicas clientelistas. Sin embargo, en los últimos años ha habido cierto acercamien-

33 Decreto Nacional 1216. 
to entre candidatos a la alcaldía y el Comité ${ }^{34}$ y entre concejales y el Comité. Para el período 1998 - 2000 se conformó una lista de candidatos al Concejo, vinculados al Comité. De ella salieron elegidos dos. Posteriormente, el Concejo contrató al Comité para elaborar el Plan de Desarrollo con participación ciudadana; de igual manera, para convalidar el Plan en reemplazo del Consejo Municipal de Planeación. Parece ser, que varios concejales, hoy, son antiguos miembros del Comité de Participación Comunitaria de Versalles.

En síntesis, la participación ciudadana en Colombia, posee un abanico de posibilidades que en gran media está sub - utilizado, en parte porque hay limitaciones estructurales como la apatía ciudadana, o vacíos que han dejado las leyes y que la frenan u obstaculizan. No obstante, se ha ido desarrollando en tres frentes. Uno débil en cultura política y pegado a las costumbres tradicionales. Otro con niveles de proyección y claridad conceptual sobre la importancia de estas opciones de inclusión político, pero que aún no logra impactar y transformar. Un tercero, a través de las organizaciones privadas: fundaciones sin ánimo de lucro con recursos de la empresa privada, las instituciones de nivel nacional y organismos internacionales que inciden en el desarrollo socioeconómico de las regiones, con programas y proyectos donde las comunidades tienen un nivel de prota- gonismo, no así los gobiernos locales o departamentales que están ausentes o son convidados de segunda mano. Esta última alternativa, sin bien genera desarrollo y como en los programas señalados, incide en las causas estructurales de la exclusión socioeconómica, deteriora la legitimidad del Estado, porque este se desentiende, y lo público tiende a concentrarse en manos de particulares privatizándose, privatizando la descentralización y reduciendo el Estado no sólo en tamaño, sino también en sus responsabilidades.

\section{Conclusiones}

Del proceso de reforma del Estado en Colombia se colige que la aplicación del paradigma mercadocéntrico y policéntrico ha propiciado a un debilitamiento del Estado a nivel subnacional. En primer lugar, ha privilegiado las exigencias de ajuste fiscal de las entidades financieras internacionales, preocupándose más por lo monetario financiero que por lo fiscalista. Como consecuencia, la reducción de las transferencias de los recursos fiscales de la nación está dejando a su suerte un significativo número de municipios que por las condiciones socioeconómicas de sus habitantes no cuentan con capacidad fiscal, para incrementar su base tributaria.

En segundo lugar, con la reducción de la burocracia se ha incrementado el

34 La información sistematizada, respecto del municipio de Versalles llega al año 2000. Se desconoce, que avances ha habido en los últimos cuatro años 
número de organizaciones privadas que contratan con el Estado la prestación de servicios públicos, muchas de ellas no ofrecen capacidad de infraestructura ni de recurso humano calificado para responder con eficiencia y eficacia las demandas sociales, principalmente aquellas de los sectores que han estado excluidos de los derechos sociales y de la acción pública. Entonces, al amparo de la contratación con el Estado se ha incrementado la irresponsabilidad, el clientelismo y el uso indebido de los dineros públicos para beneficio privado.

Y el paradigma policéntrico mediante el cual se promueve la socialización política de los sectores excluídos de la participación en los asuntos del Estado también muestra tendencias a la privatización, aunque hay expresiones ciudadanas como la del municipio de Versalles o las que jalonan organizaciones universitarias o fundacionales sin ánimo de lucro que tienen trayectoria en ejercicios de control social y que buscan como objetivo, estimular e incentivar dinámicas participativas y cambios en el comportamiento político de la ciudadanía. La labor de organizaciones para el desarrollo como promotoras de la participación es también un esfuerzo por fortalecer el tejido social, pero se resisten a relacionarse con los gobiernos locales o departamentales generando paralelismos que deterioran cada vez más la credibilidad y legitimidad del Estado, el que pareciera, estar condenado a ser esquilmado directa o indirectamente para la repro- ducción de quienes no quieren abandonar la cultura política tradicional.

\section{Referencias bibliográficas}

BORDA, Jordi y otros. "Descentralización del Estado, movimiento social y gestión local". pgs. 23 - 56. ICI/FLACSO/CLASCO. Santiago de Chile. (1987).

CÁRDENES Rivera, MIGUEL Eduardo. (compilador). "La reforma política del Estado en Colombia: una salida integral a la crisis". Fondo editorial CEREC, Bogotá. (2005).

CASTRO, Jaime, " $¿$ Transferencias $=$ déficit fiscal? ¿Regiones o departamentos? Corporación Viva la Ciudadanía" - Federación Nacional de Concejales, Foro Nacional por Colombia, Fundación Fescol. Bogotá. (2001).

CEBALLOS, Miguel, GERALD Martín. "Participación y fortalecimiento institucional a nivel local en Colombia". Centro Editorial Javeriano. Pontificia Universidad Javeriana, Bogotá. (2001).

Código de Régimen Municipal. Editorial Oveja Negra. Bogotá, $2^{\mathrm{a}}$ edición. (1986).

Constitución Política de Colombia. Editorial Temis. Santafé de Bogotá. (1991).

CORAGGIO, José Luis. "Descentralización, el día después..." UBA, Buenos Aires, pg. 9 - 37. (1997).

CUNILL, Nuria. "Dilemas y perspectivas para la democratización de los Estados latinoamericanos". Capítulo 1. En el Discurso teórico relativo a la Participación Ciudadana. CLAD. Caracas. (1991).

Departamento Nacional de Planeación. Capacidad Fiscal de los gobiernos territoriales colombianos. Dirección de Desarrollo Territorial Sostenible. Bogotá, Abril de 2005. www.dnp.gov.co. 
GONZÁLEZ Salas, Edgar Alfonso “El sistema de transferencias integubernamentales". En descentralización y Estado Moderno. Fundación Fescol. Bogotá, pgs. 23 - 61. (1991).

JARAMILLO, Iván. "El recorte de las transferencias a las entidades territoriales y las políticas de saneamiento fiscal. En $¿$ Transferencias $=$ déficit fiscal?. ¿Regiones o departamentos? Corporación Viva la Ciudadanía" - Federación Nacional de Concejales, Foro Nacional por Colombia, Fundación Fescol. Bogotá. (2001)

MENY, Ives. JEAN-CLAUDE Thoenig. "Las políticas públicas. Ariel Ciencia Política”. Barcelona. (1989)

STEWART, Frances. "Todos entran. Propuesta para sociedades incluyentes". Editorial Santillana, Bogotá Colombia, pg. $25-65$. (1989)
VARELA, B. Edgar . "La mercantilización de lo público". En Revista Instituciones y Desarrollo No. 14-15. (2003). http://www.iigov.org.

VELÁSQUEZ, Fabio. "La veeduría ciudadana en Colombia: en busca de nuevas relaciones entre el Estado y la sociedad civil". En Bresser Pereira, Luis Carlos y Cunill Grau, Nuria (editores), Lo Público no estatal en la reforma del Estado, Buenos Aires, CLAD/Paidós.(1998).

VELÁSQUEZ, Fabio. GONZÁLEZ, Esperanza. "¿Qué ha pasado con la participación ciudadana en Colombia?". Bogotá, Fundación Corona. (2003)

WIESNER, Eduardo. WIESNER D, Eduardo. "La efectividad de las políticas públicas en Colombia. Un análisis neoinstitucional". Tercer mundo editores. Bogotá, 1998. 


\title{
RESÚMENES Y ABSTRACTS
}

\section{LOS VALORES EN LAS FAMILIAS EMPRESARIAS COLOMBIANAS}

\author{
Ernesto Barrera Duque
}

\section{RESUMEN}

Este artículo, a partir de una investigación cualitativa y práctica, propone un modelo general de análisis para los valores en las dinámicas operativas de las familias empresarias colombianas. En el cuerpo del texto, se identifican los valores vigentes, estableciéndose su contenido y jerarquía, en función de los resultados del trabajo de campo.

PALABRAS CLAVE: Familia empresaria, empresa familiar, valores.

\begin{abstract}
This article, from a qualitative and practical research, proposes a general model of analysis for the dynamic values in operative the families of the Colombian industralists. In the body of the text, the effective values are identified, settling down their content and hierarchy, based on the results of the work of field.
\end{abstract}

KEY WORDS: Family entrepreneur, familiar company, values. 


\title{
ANÁLISIS DEL DISEÑO DE LOS CONTRATOS EN EL PROCESO DE SUBCONTRATACIÓN DE LAS ORGANIZACIONES PÚBLICAS, EL CASO DE EMCALI EICE
}

\author{
Henry Caicedo Asprilla
}

\begin{abstract}
RESUMEN
Éste documento utiliza las categorías de la economía institucional para evaluar el impacto que tiene sobre las organizaciones públicas el proceso de outsourcing o subcontratación. Aquí se plantea el interrogante de hasta dónde es posible alcanzar la eficiencia de una organización pública cuando el proceso de subcontratación está determinado por contratos intrínsecamente incompletos. A partir de la información de una muestra de 198 contratos que Emcali firmó con proveedores entre los años 2001 y 2004, se halló que 128 estaban mal diseñados y sólo 70 cumplían con el requisito del buen diseño económico; este hallazgo permite verificar la presunción de la teoría económica institucional que afirma que aunque los contratos estén diseñados conforme a la ley, estos presentan fallas en el diseño económico y por consiguiente la empresa experimenta costos de transacción. En este docu-
\end{abstract}

mento se evidencia que en el caso concreto de Emcali existen dos variables que clasifican los contratos entre bien y mal diseñados: el valor de la inversión y el índice de riesgo de ejecución del contrato; mientras que las variables duración del contrato, el riesgo legal, empresas en licitación, garantías y pólizas de aseguramiento, consideradas fundamentales en el diseño contractual de una transacción no cumplen con el propósito de corregir las anomalías en los contratos. En este trabajo se avanza hasta plantear que existe una conexión entre el mal diseño de los contratos y las dificultades financieras y administrativas de Emcali, que llevó a la empresa a la crisis más grande de su historia.

PALABRAS CLAVE: Organizaciones públicas, outsourcing, análisis de los contratos, economía institucional, árboles de decisión. 


\section{ABSTRACT}

This paper uses the categories of the institutional economy to evaluate the impact that has on the public organizations the process of outsourcing or subhiring. The principal question is: until where considers it is possible to reach the efficiency of a public organization when the subhiring process is determined by intrinsically incomplete contracts. From the information of a sample of 198 contracts that Emcali signed whit suppliers between years 2001 and 2004, one was that 128 badly were designed and only 70 fulfilled the requirement of the good economic design, this finding allows to verify the economic presumption of the institutional theory that it affirms that although the contracts are designed according to the law, these contracts present faults in the economic design and therefore the company experiences transaction costs. This document evidences that in the Emcali's case there are two indicator that classify contracts between good and badly designed: the value of the investment and the index of risk of execution of the contract, whereas the indicators duration of the contact, the legal risk, companies in licitation, guarantees and insurance policies, were considered fundamental in the contractual design of a transaction do not correct the anomalies of the contacts. In this paper one advances when a connection is established between the wrong design of contracts and the financial and administrative difficulties of Emcali, that led the company to the greatest crisis of its history.

KEY WORDS: Public organizations, outsourcing, analysis of contracts, institutional economy, decition trees. 


\title{
¿DESDE UNA INGENIERÍA DEL ALMA HACIA UN "MANAGEMENT" DEL ESPÍRITU? ACERCA DE LA NOCIÓN DE PSICOLOGÍA EN EL DISCURSO ADMINISTRATIVO Y ORGANIZACIONAL
}

\author{
Héctor Bermúdez
}

\section{RESUMEN}

Este ensayo busca un acercamiento a la genealogía de lo que se reconoce en la administración como las "Ciencias del Comportamiento" y una crítica a la relación entre el pensamiento administrativo y aquella corriente particular de la psicología que ha sido incorporada a éste: lo que comúnmente se conoce como la psicología industrial pero que bien podría nombrarse como el conductismo organizacional.

Cabe decirse también, que, por el trabajo de consultoría especializada en Gestión Humana del autor, y por la docencia tanto en cursos de MBA para ejecutivos, como en programas para especialistas en administración de personal, fue posible articular esa experiencia de campo, con lo inferido del análisis teórico de la revisión documental. Así entonces, este trabajo busca apoyar la línea de investigación en estudios sobre la Gestión Humana.

PALABRAS CLAVE: Administración, Conductismo Organizacional, Psicoanálisis, Psicología Industrial, Organizaciones.

\begin{abstract}
This test looks for an approach of the genealogy of which a critic is recognized in the administration like "Sciences of the Behaviour" on the other hand, the article explain the relation between the administrative thought and that particular current of the psychology that has been incorporated to this one. This challenge is commonly known like the industrial psychology but that could well name like the organizational conductism.

It is possible also to say itself, that, by the work of consultancy specialized in Human Management of the author, and by teaching as much in courses of MBA for executives, like in programs for specialists in personnel administration, it was possible to articulate that experience of field, with the inferred thing of the theoretical analysis of the documentary revision. Then, this work looks for to support the line of research in studies on the Human Management.
\end{abstract}

KEY WORDS: Administration, Organizational Conductism, Psychoanalysis, Industrial Psychology, Organizations. 


\title{
LOS DESAFÍOS DEL DISEÑO ORGANIZACIONAL E INSTITUCIONAL EN UN MEDIO AMBIENTE GLOBALIZADOR
}

\author{
José G. Vargas Hernández
}

\section{RESUMEN}

Este trabajo tiene por objetivo analizar los desafíos de diseño organizacional e institucional en un medio ambiente globalizador. Este análisis se inicia a partir de las consideraciones de los procesos de globalización económica en la emergencia de las nuevas asimetrías del poder y su impacto en los procesos de cambio organizacional, institucional, del Estado y las políticas públicas y el diseño intergubernamental. La información y el conocimiento son insumos de las comunidades de aprendizaje y para la formación de redes. Una característica de los arreglos institucionales y nuevas formas organizacionales es la creación del poder en los individuos (empowerment)

PALABRAS CLAVE: Asimetrías del poder, diseño institucional, diseño organizacional, globalización

\begin{abstract}
This work must by objective analyze the challenges of organizational and institutional design in global environment. This analysis begins from the considerations of the processes of economic globalization in the emergency of the new asymmetries of the power and its impact in the processes of organizational, institutional change, of the State and the public policies and the intergovernment design. The information and the knowledge are raw material of the learning communities for the formation of networks. A characteristic of the institutional adjustments and new organizationals forms is the creation of the power in the individuals (empowerment)
\end{abstract}

KEY WORDS: Asymmetries of the power, institutional design, organizational design, globalization. 


\title{
LA REFORMA DEL ESTADO EN COLOMBIA: ENTRE EL AJUSTE FISCAL Y FORTALECIMIENTO DE LA PARTICIPACIÓN CIUDADANA
}

\author{
Rosalía Correa Young
}

\begin{abstract}
RESUMEN
La reforma del Estado en Colombia y la descentralización, desde que se aprobó el Acto legislativo 01 de 1986, pasando por los acuerdos institucionales, plasmados en la Constitución de 1991 y referidos a esta materia, así como la normatividad actual, son analizados en este artículo a la luz de los enfoques teóricos que estimularon un nuevo modelo de Estado, a partir de la percepción respecto del agotamiento del Estado de bienestar, interventor y asistencial.

El artículo explica la dinámica en Colombia frente a dos paradigmas: uno proveniente de la sociología y la ciencia política que define la reforma desde una perspectiva policéntrica y uno proveniente de la economía neoclásica que ofrece una perspectiva mercadocéntrica. La primera en-
\end{abstract}

cuentra fortalezas para la democratización, la equidad, la superación de los desequilibrios regionales y la inclusión social. La segunda, el posicionamiento de las lógicas del mercado con la reducción, o desaparición de la intervención del Estado en aquellas funciones que los privados pueden resolver más eficientemente. Uno y otro colocan a la sociedad civil en un protagonismo activo, sea político, en su relación con el Estado; sea económico, en su relación con el mercado, pero ambos la conciben como actor de primera línea.

PALABRAS CLAVE: Reforma del Estado, Descentralización, Democratización, Paradigma policéntrico, Paradigma mercadocéntrico, Ajuste Fiscal, Participación, Privatización 


\section{ABSTRACTS}

The reform of the state in Colombia and the decentralization, since legislative Act 01 of 1986 was approved, happening through the agreements institutional, shaped in the Constitution of 1991 and referred this matter, as well as the present normatividad, are analyzed in this article to the light of the theoretical approaches that stimulated a new model of state, from the perception respect to the exhaustion of the welfare state, auditor and healthcare.

The article explains dynamics in Colombia as opposed to two paradigms: one originating of sociology and political science that the reform defines from a policentric perspective and one originating of the neoclassic economy that offers a market perspective. First it finds strengths for the democratization, the fairness, the overcoming of the regional imbalances and the social inclusion. Second, the positioning of the logics of the market with the reduction, or disappearance of the intervention of the State in those functions that the prevailed ones can solve more efficiently. Both places to the civil society in an active protagonism, is politician, in their relation with the State; be economic, in his relation with the market, but both conceive it like actor of forward edge.

KEY WORDS: The reform of the State, Decentralization, Democratization, policentric Paradigm, market Paradigm, Fiscal Adjustment, Participation, Privatization. 


\section{TEORÍA ECONÓMICA Y ESTADO DEL BIENESTAR. UNA APROXIMACIÓN}

Fernando Salazar Silva

\section{RESUMEN}

El siguiente artículo realiza un tránsito por algunas posturas dentro de las ciencias económicas para discutir el EB y presentar alternativas concretas al mismo. El propósito de Keynes en su afán de hacer legitimar la configuración y consolidación del Estado de Bienestar, se discutirá en el contexto de las particularidades de carácter teórico económico. Si bien el keynesianismo pudo concretar desde las ciencias económicas las bases para acercar la teoría a la realidad social, no entendemos por qué otras escuelas no han logrado constituirse en la base teórica del Estado de Bienestar actual.

PALABRAS ClAVE: Estado de Bienestar, Pacto keynesiano, Ciencia económica, Monetarismo, Expectativas racionales, teoría Neoclásica, Neokeynesianismo.

\begin{abstract}
The following article makes a transit by some positions within economic sciences to discuss the EB and to present concrete alternatives to he himself. Keynes's intention in its eagerness to make legitimize to the configuration and consolidation of the Welfare State, will be discussed in the context of the particularities of economic theoretical character. Although the keynesianism could make specific from economic sciences the bases to approach the theory the social reality, we do not understand so that other schools have not managed to constitute themselves in the theoretical base of the Welfare State present.

KEY WORDS:

Welfare State, Keynesian Accord, Economic Science, Monetarism, Rational Expectations, Theorie Neoclassic, Neo-Keynesianism.
\end{abstract}




\title{
LA RESPONSABILIDAD CIVIL AUTOMOVILÍSTICA Y EL ASEGURAMIENTO OBLIGATORIO Y VOLUNTARIO: UN ESTUDIO EN EL DERECHO COLOMBIANO Y ESPAÑOL
}

\author{
Raquel Ceballos Molano
}

\begin{abstract}
RESUMEN
La accidentalidad vial en Colombia es una de las primeras causas de daños corporales y materiales en la población circulante y peatonal, que obliga, al menos en teoría, al propietario o conductor, a responder civilmente de sus consecuencias. Así, este trabajo de tesis plantea el problema de la tipicidad como "actividad peligrosa" y la dispersión normativa derivada de la responsabilidad civil automovilística y los mecanismos de reparación a través del seguro voluntario y obligatorio del automóvil, consagrada en la legislación Civil, Penal, Comercial, Código Nacional de Tránsito, y en Decretos y Resoluciones administrativas, además de la doctrina científica y jurisprudencial, lo que evidencia que el legislativo colombiano no ha prestado atención, desde el punto de vista técnico y jurídico a las políticas de Estado para armonizar en un solo estatuto la responsabilidad derivada de los accidentes de tránsito y su aseguramiento, como si ocurre en el derecho comparado, el ordenamiento jurídico europeo y español.
\end{abstract}

PALABRAS CLAVE: Accidente de transito, responsabilidad civil, seguro obligatorio, soat, seguro automoviles, daño, indemnizacion, reparacion integral.

\section{ABSTRACT}

The road crash in Colombia is one of the first causes of body and materials damages in the circulating and peatonal population, that forces, at least in theory, to the proprietor or conductor, to respond civilly of its consequences. Thus, this thesis work creates the problem of the type like "dangerous activity" and the normative dispersion derived from the automobile civil responsibility and the mechanisms of repair through voluntary and obligatory insurance of the automobile, consecrated in the Civil, Penal, Commercial legislation, National Code of Transit, and in Decrees and administrative Resolutions, in addition to the scientific and jurisprudencial doctrine, which evidence that the legislative Colombian has not paid attention, from the technical and legal point of view to the state policies to harmonize in a single statute the responsibility derived from the traffic accidents and its securing, as if happens in the compared right, the European and Spanish legal ordering.

KEY WORDS: Road accident, civil responsibility, surely obligatory, soat, surely automobiles, damage, indemnification, integral repair. 


\title{
ENVEJECIMIENTO DEL PERSONAL
}

\author{
Sergio Castrillón
}

\section{RESUMEN}

¿De que manera la literatura en ciencias administrativas ha abordado el estudio del envejecimiento del personal? ¿Cuáles son las razones que han motivado este interés en los últimos años? Cuáles son las lógicas subyacentes? Reconociendo las tendencias demográficas mundiales que reflejan el envejecimiento de la población; este artículo procura responder a esta preguntas, a partir de una revisión exhaustiva de las principales publicaciones que se han ocupado del tema en el contexto internacional. Nuestro propósito es aumentar la visibilidad de la problemática y contribuir al debate sugiriendo acciones que revaloricen su dimensión humana.

PALABRAS CLAVE: Administración - Envejecimiento del Personal - Recursos Humanos - Fuerza Laboral - Jubilación - Retiro - Competencias y Desempeño - Motivación - Entrenamiento

\section{ABSTRACT}

How Literature in administrative sciences is boarded the study of the ageing of the personnel? Which are the reasons that have motivated east interest in the last years? Which are the underlying logics? Recognizing world-wide demographic tendencies that reflect the ageing of the population; this article tries to respond to this questions, from an exhaustive revision of the main publications that have taken care of the subject in the international context. Our intention is to increase the visibility of the problematic one and to contribute to the debate being suggested actions that revalue their human dimension.

KEY WORDS: Administration Ageing of the Personnel - Human Resources -Labor Force - Retirement - Competitions and Performance - Motivation - Training 


\title{
RENDICIÓN DE CUENTAS: ESTRATEGIA PARA EL FORTALECIMIENTO DE LA DEMOCRACIA A TRAVÉS DE LA LIMITACIÓN DEL PODER
}

\author{
Carlos Wladimir Gómez Cárdena
}

\begin{abstract}
RESUMEN
Este artículo contiene como apuesta epistemológica la construcción de un modelo genérico de Rendición de Cuentas, el cual puede llegar a ser implementado en las diferentes entidades de naturaleza estatal. A partir de una elaboración teórico - conceptual de la Rendición de Cuentas y de una observación práctica de los primeros ejercicios de rendición de cuentas llevados a cabo en Colombia, se exploran diversas potencialidades e instrumentos que pueden llegar a fortalecer las democracias modernas a través fundamentalmente, de la limitación de los ejercicios de poder. En las democracias modernas son muchos los mecanismos formales de limitación del poder consignados en el conocido sistema de frenos y contrapesos, pero son muy pocos los mecanismos reales. La rendición de cuentas como práctica democrática puede presentarse como uno de estos últimos, en la medida en que la sociedad organizada explore y explote tales potencialidades.
\end{abstract}

PALABRAS CLAVE: Políticas Públicas / Administración Pública / Rendición de Cuentas / Democracia / Limitación del Poder.

\section{ABSTRACT}

This article contains like comely epistemological the construction of a generic model of Accountability, which can arrive to be implemented in the different organizations of state nature. Starting from a theoretical - conceptual elaboration of the Accountability and of a practical observation of the first exercises of Accountability carried out in Colombia, diverse potentialities and instruments are explored that can end up strengthening the modern democracies fundamentally to inclination, of the limitation of the exercises of power. In the modern democracies there are many formal mechanisms of limitation of the power consigned in the well-known system of check and balances, but there are very few real mechanisms. Accountability as democratic practice can be presented like one of these last ones, in the measure in that the organized society explores and exploit such potentialities.

KEY WORDS: Public Policies / Public Administration / Accountability / Democracy / Limitation of the Power. 